\title{
ORIGINAL ARTICLE \\ Spinal cord injury resulting from gunshot wounds: a comparative study with non-gunshot causes
}

\author{
Ü Güzelküçük, Y Demir, S Kesikburun, B Aras, F Yavuz, E Yaşar and B Yılmaz
}

Study design: Retrospective, comparative 7-year study.

Objectives: To identify the clinical characteristics of patients with spinal cord injury (SCI) resulting from gunshot wound (GSW).

Setting: Turkish Armed Forces Rehabilitation Center, Ankara, Turkey.

Methods: The study included 1043 consecutive patients with SCl who were divided into two groups according to etiology: patients with gunshot-induced spinal cord injury (GSWSCI) constituted the study group, and randomly selected patients with non-gunshot-induced spinal cord injury (NGSWSCI) who were matched for gender and for week of admission constituted the control group. The demographic and clinical characteristics of the patients were recorded, compared and analyzed.

Results: The study group included 102 patients (mean age: $26.93 \pm 9.11$ years). The vast majority of the patients were aged $16-30$ years (68.6\%) and $90.2 \%$ were male. The majority of the lesions were at the thoracic level (58.8\%) and a complete injury (60.8\%). Surgical stabilization of the spine was performed in 50 patients (49\%). The most prevalent associated injury was intra-abdominal injury followed by chest injury. Compared with the NGSWSCI group, the GSWSCI patients were more likely to have a complete lesion (60.8\% vs $45.1 \%, P=0.025$ ), had a lower rate of surgical stabilization (49 vs $88.2 \%, P=0.0001$ ) and had a higher rate of associated injuries (54.9\% vs $25.5 \%, P=0.0001$ ). Compared with the civilian GSWSCI group, the military GSWSCl patients had a higher rate of surgical stabilization and associated injuries (60\% vs $40 \%, P=0.049,68.9 \%$ vs $43.9 \%, P=0.012$, respectively).

Conclusion: The results revealed that GSWSCI and military GSWSCI patients may have different demographic and clinical features compared with NGSWSCI and civilian GSWSCI patients, respectively.

Spinal Cord (2016) 54, 737-741; doi:10.1038/sc.2016.29; published online 1 March 2016

\section{INTRODUCTION}

Spinal cord injury (SCI) is one of the potentially catastrophic consequences of gunshot wounds (GSWs). Gunshot-induced spinal cord injury (GSWSCI) has become increasingly important with the rise in the number of civilian-owned guns, violence in urban areas and conflicts in many parts of the world. GSWs are categorized according to the energy transfer characteristics along the missile path. ${ }^{1}$ Civilian GSWs tend to result from small arms and light weapons with low velocity, but high velocity GSWs generally occur in the military population. $^{2,3}$

SCI caused by GSWs is a complex injury and develops through different mechanisms including the direct impact of the bullet, the pressure of shock waves and temporary cavitation. ${ }^{4-7}$ Civilian spinal GSWs have different ballistic characteristics, with damage mainly caused by a direct mass effect. ${ }^{6}$ The extent of tissue damage correlates with the distance between the gun and target, trajectory, size, shape and velocity of the bullets. ${ }^{5,7-9}$ GSWs from military-type weapons with high velocity have recently increased in the civilian population. ${ }^{2}$ Therefore, the findings of studies on GSWSCI performed a few decades ago may differ from those of current studies.

Previously, studies of GSWSCI have been primarily based on either a military or a civilian population. The primary purpose of the present study was to identify the clinical and demographic characteristics of
SCI patients resulting from GSW. The secondary purpose was to compare SCI of military and civilian GSW and to determine the difference between GSWSCI and non-gunshot-induced spinal cord injury (NGSWSCI).

\section{MATERIALS AND METHODS}

The medical records were reviewed of 1043 patients with traumatic SCI admitted to the Turkish Armed Forces (TAF) Rehabilitation Center, Ankara, Turkey, during a 7-year period (January 2007-December 2013).

Patients with SCI were divided into two groups according to the etiology: patients with GSWSCI constituted the study group, and randomly selected patients with NGSWSCI who were matched for gender and week of admission constituted the control group (Figure 1). In addition, patients with GSWSCI were divided into two subgroups of civilian or military. Patients with SCI resulting from explosive mechanisms (shrapnel, mines etc.) were excluded from the study.

Demographic and clinical data including age, gender, etiology, time since injury, degree and level of neurological impairment, length of rehabilitation stay, bladder management methods, surgical stabilization including vertebral arthrodesis, spinal instrumentation and decompression, associated injuries and complications were recorded. The patients were divided into six age groups based on age at the time of injury: $0-15$ years, $16-30$ years, $31-45$ years, $46-60$ years, $61-75$ years and $\geqslant 76$ years.

The extent of the injury was classified based on the ASIA-ISCoS International Standard of Neurological Classification of Spinal Cord Injury 


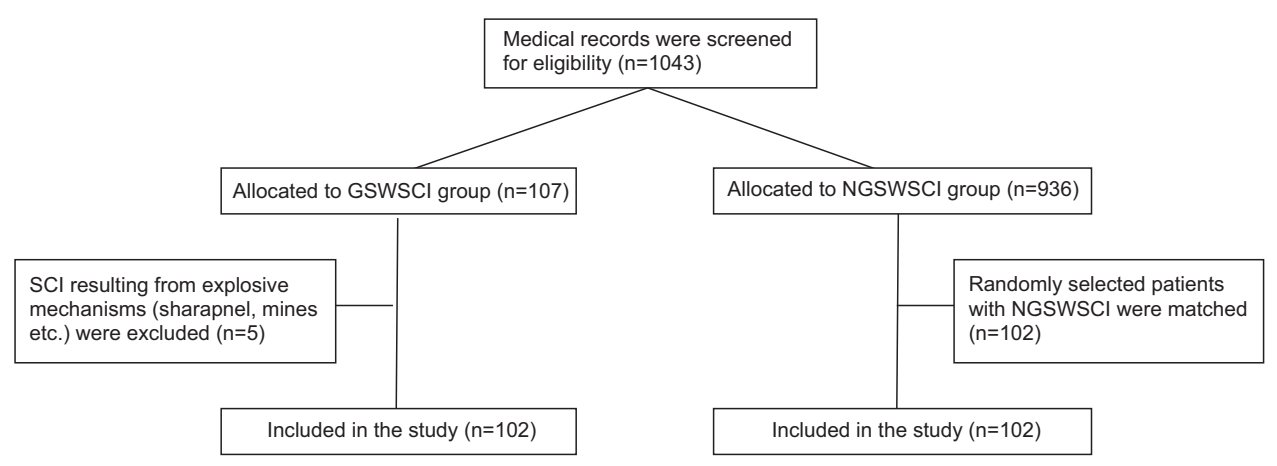

Figure 1 Study methodology flow chart.

(http://www.iscos.org.uk/). The level of injury was classified as cervical, thoracic or the lumbar region. This study protocol was approved by the Gülhane Military Medical Academy Ethics Committee.

Statistical analysis was performed using SPSS v.15.0 for Windows (SPSS, Inc., Chicago, IL, USA). Continuous variables are presented as mean \pm s.d. and range (min.-max.), and categorical variables are shown as frequency and percentage. The $\chi^{2}$-test was used to determine the level of significance of the difference in nominal variables between groups. The normality of the distribution of numeric variables was examined using the Kolmogorov-Smirnov test. Differences between groups were determined via the Mann-Whitney $\mathrm{U}$ test. The level of statistical significance was defined as $P<0.05$.

\section{RESULTS}

During the 7-year study period, 102 (9.8\%) GSWSCI (study group) were identified from a total of 1043 traumatic SCI patients. Of the 102 patients with GSWSCI included in the study, 57 (55.9\%) sustained civilian GSWSCI, whereas 45 sustained military GSWSCI. The control group comprises 102 of the NGSWSCI patients admitted during the study period. The demographic and clinical characteristics of the study and control groups are shown in Table 1.

In the study group, 90.2\% were male (male-female ratio: 9.2:1). Mean age at the time of injury was $26.93 \pm 9.11$ years (range: $13-52$ years). Most of the patients were aged 16-30 years $(n=70,68.6 \%)$, followed by $31-45$ years $(n=20,19.6 \%)$. The most common level of injury was the thoracic region $(58.8 \%)$ followed by cervical $(21.6 \%)$ and lumbosacral $(19.6 \%)$. The severity of SCI was determined as complete injury in $60.8 \%$ of the patients and incomplete injury in $39.2 \%$. Complete paraplegia was the most frequent neurological category of the patients (46\%). The most common bladder management method was clean intermittent catheterization (65.7\%). Surgical stabilization of the spine was performed in 50 patients (49\%). The severity of the injury was similar in surgical and conservative management (Figure 2). Of the 102 patients with GSWSCI, 30 $(29.4 \%)$ had an intra-abdominal injury including the intestines, liver and spleen, $26(25.5 \%)$ had a chest injury, $14(13.7 \%)$ had $\geqslant 1$ upper or lower extremity fractures and $2(2 \%)$ had other injuries (head and aortic injury). The findings of the control group are presented in Table 1 .

The mean age of the GSWSCI patients was lower than that of the NGSWSCI patients $(P=0.01)$. Compared with the NGSWSCI patients, the GSWSCI patients were more likely to have a complete lesion $(60.8 \%$ vs $45.1 \%, P=0.025)$. GSWSCI patients had a lower rate of surgical stabilization and a higher rate of associated injuries than those with NGSWSCI ( $49 \%$ vs $88.2 \%, P=0.0001$ and $54.9 \%$ vs $25.5 \%$, $P=0.0001$, respectively). The level of SCI and bladder management methods were similar in both groups $(P>0.05)$. Upper thoracic
(T1-6) SCI was more common in the GSWSCI group than in the NGSWSCI group ( $n=28 / 60,46.7$ vs $n=11 / 54,20.4 \%, P=0.003)$. In addition, there was a significant difference in the severity of injury at the thoracic level $(P=0.017)$ (Table 2).

The mean age at the time of injury was $23.13 \pm 4.04$ years and $29.93 \pm 10.77$ years for the military GSWSCI and civilian GSWSCI groups, respectively. There were significant differences in both mean age and the age group between the military and civilian GSWSCI groups $(P=0.0001)$. All of the military GSWSCI patients were male, whereas $82.5 \%$ of the civilian GSWSCI group was male. Compared with the civilian GSWSCI group, the military GSWSCI group had a higher rate of surgical stabilization and associated injuries (60\% vs $40 \%, P=0.049,68.9 \%$ vs $43.9 \%, P=0.012$, respectively). There was a significant difference in the level of SCI between the two groups $(P=0.034)$.

\section{DISCUSSION}

Traditionally, the leading causes of SCI are motor vehicle accident and falls. ${ }^{10-13}$ Although the proportion of these etiologies is almost similar in different countries, GSWSCI, which has an important place in the etiology of SCI, has a rather wide range of proportion among all causes of SCI. In some countries, GSWSCI exceeds motor vehicle accident or falls, whereas it is considerably lower in others. The rates of GSWSCI have been reported as $25.8 \%$ in Jordan, $11.6 \%$ in Brazil, $1.6 \%$ in Russia, $7.3 \%$ in Nigeria and $10.4 \%$ in USA. ${ }^{10,12}$ The geographic location, level of development and gun control laws of the countries seem to have especially significant roles in the incidence of GSWSCI. An earlier study performed in a region of Turkey between 1990 and 1999 found that the proportion of GSWSCI was $21.3 \% .^{13}$ A study conducted at our Center between 2000 and 2007 revealed that the proportion of GSWSCI was $12.2 \%$, as the third most common cause of SCI. ${ }^{11}$ In the present study, patients with GSWSCI accounted for $9.8 \%(102 / 1043)$ of all admissions during the study period. In comparison with the past in Turkey, the results of the current study show a reduction in the rates of GSWSCI, which may be due to stricter weapon control laws and a decreased level of conflict and civil unrest during the study period.

GSWSCI is of great importance because it mainly affects a young population with the mean age of patients ranging from 25 to 28.8 years. ${ }^{4,6,14-16}$ Chen et al. ${ }^{10}$ reported that, although GSWSCI was the third most common cause of all SCI, it was the second in the 16-30-year age group and then declined rapidly with advancing age. In the present study, the mean age was 26.9 years, and the 16-30-year age group was found to be at consistently higher risk of GSWSCI $(68.6 \%)$. In addition, the results revealed that the mean age of patients with GSWSCI was lower than those with NGSWSCI; hence, this result 
Table 1 Demographic and clinical characteristics of the patient groups

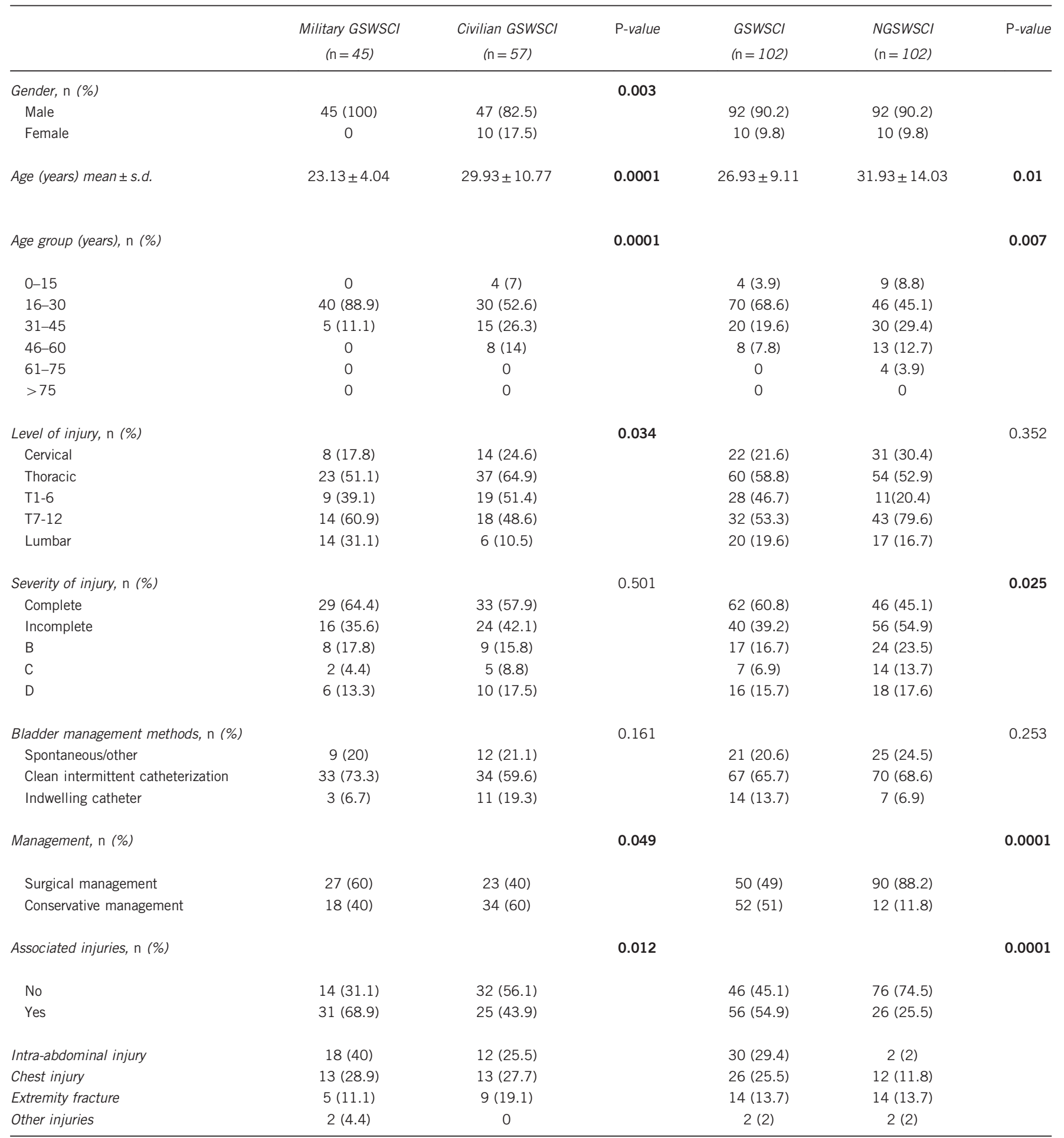

Abbreviations: GSWSCI, gunshot-induced spinal cord injury; NGSWSCI, non-gunshot-induced spinal cord injury; s.d., standard deviation.

Values in bold indicate statistically significant differences at $5 \%$.

appears to be related to military patients. However, the civilian GSWSCI and NGSWSCI groups were similar in age (29.93 vs 31.93 years).

The proportion of male patients with GSWSCI has been reported to range from 77.5 to $94 \% .4,6,10,11,14,15,17$ Chen et al. ${ }^{10}$ reported NGSWSCI in males at almost $77.2 \%$ and GSWSCI at $87.9 \%$.
In the present study, GSWSCI in males was almost nine times higher than in females. Not surprisingly, these results indicate that male predominance was more obvious in GSWSCI.

The level and severity of SCI are associated with the etiology of the injury. ${ }^{10}$ Chen et al. ${ }^{10}$ reported that GSWSCI typically resulted in paraplegia (71.8\%), and NGSWSCI almost always resulted in 


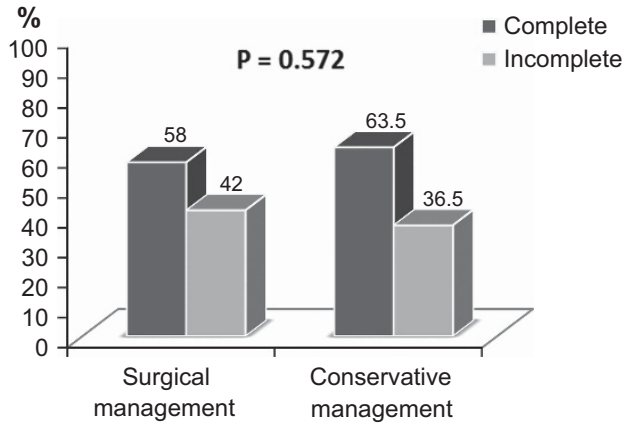

Figure 2 Injury severity of GSWSCI according to the management method.

tetraplegia (61.8\%). In the current study, tetraplegia was less common in the GSWSCI group than in the NGSWSCI group (21.6\% vs $30.4 \%$ ). The low rate of tetraplegia may be due to high-mortality rates because of the presence of vital structures and due to the small target area in cervical GSWs compared with other levels. Interestingly, the civilian GSWSCI patients had higher rates of tetraplegia than the military GSWSCI patients (24.6\% vs $17.8 \%)$. Alaca et al. ${ }^{9}$ reported only $1.9 \%$ tetraplegia in military GSWSCI cases. These results may be associated with a high rate of civilian GSWSCI inflicted at close range. The most common site of GSWSCI is the thoracic level, which is in agreement with the present findings. ${ }^{8,16,17}$ Although GSWSCI patients had a low rate of cervical SCI, upper thoracic SCI (T1-6) was more common than in the NGSWSCI group (46.7\% vs $20.4 \%$ ).

Compared with the NGSWSCI group, the GSWSCI patients were found to be more likely to have a complete lesion (60.8\% vs $45.1 \%)$, which is consistent with the findings of previous studies. ${ }^{10,16}$ The rate of complete injury was highest at the thoracic level (75\%), in line with a study performed by Trahan et al. ${ }^{15}(66 \%)$. Completeness of injury may be related to the anatomic region. That the structure of the thoracic level is more stable than the cervical and lumbar levels may make it more vulnerable to GSW. The most common type of GSWSCI was complete paraplegia (46\%), as also observed in previous studies. ${ }^{9,14}$ The velocity of the bullet is the most important determinant of the severity of the SCI. ${ }^{3}$ As expected, military weapons with high velocity are more destructive than small arms and light weapons with low velocity. Alaca et al. ${ }^{9}$ reported $84 \%$ complete injury in military GSWSCI cases. In the present study, military GSWSCI patients were more likely to have a complete lesion when compared with civilian GSWSCI patients $(64.4 \%$ vs $57.9 \%)$, but the difference was not significant.

Le Roux and Dunn ${ }^{17}$ reported that stabilization was performed in only $12.2 \%$ of unstable GSWSCI cases. Alaca et al. ${ }^{9}$ reported that $53 \%$ of military GSWSCI patients underwent spinal surgical procedures. In the present study, although the majority of the NGSWSCI group underwent a surgical intervention for the spine $(88.2 \%)$, it was performed on only about half of the GSWSCI group (49\%). There was also a significant difference in surgical intervention between military and civilian GSWSCI cases (60\% vs $40 \%)$. These results demonstrate that the integrity of the spinal column generally remains intact in GSWSCI, especially in civilian cases. In patients who underwent surgical intervention, a complete lesion was less common than in patients who did not, but the difference was not significant. This result supports the view that surgical management for GSWSCI is not widely advocated and is controversial. ${ }^{3,8}$

The velocity of the bullets correlates with the magnitude of tissue injury and tissue loss. ${ }^{1}$ High-energy GSWs are associated with
Table 2 Severity of the injury according to the level of the injury

\begin{tabular}{lrrrrr}
\hline & \multicolumn{2}{c}{ GSWSCI } & & NGSWSCI & P-value \\
\cline { 2 - 4 } & \multicolumn{4}{c}{ Severity of injury, n (\%) } \\
\cline { 2 - 4 } & Complete & Incomplete & Complete & Incomplete \\
\hline Level of injury & & & & & \\
Cervical & $15(68)$ & $7(32)$ & $14(45.2)$ & $17(54.8)$ & 0.097 \\
Thoracic & $45(75)$ & $15(25)$ & $29(53.7)$ & $25(46.3)$ & 0.017 \\
Lumbar & $2(10)$ & $18(90)$ & $3(17.6)$ & $14(82.4)$ & 0.498 \\
\hline
\end{tabular}

Abbreviations: GSWSCI, gunshot-induced spinal cord injury; NGSWSCI, non-gunshot-induced spinal cord injury.

Value in bold indicates statistically significant difference at $5 \%$.

significantly more soft tissue devitalization and a larger zone of injury. ${ }^{18}$ Le Roux and Dunn ${ }^{17}$ reported 55 significant associated injuries in 49 GSWSCI cases. In the current study, the rates of associated injury were higher in the GSWSCI and military GSWSCI groups than in the NGSWSCI and civilian GSWSCI groups. The most common associated injuries were intra-abdominal and chest injuries, in line with previous studies. ${ }^{6,17}$ These results show that SCIs resulting from GSWs, especially military case, have an increased risk of associated injuries.

The strength of this study was the inclusion of a control group and that the study evaluated both military and civilian GSWSCI. However, there were several limitations, primarily the retrospective and single-center design. Second, the sample size was relatively small to detect possible differences between the groups. Finally, there was no exact information available as to whether the weapon was high velocity or low velocity.

The cases of GSWSCI and NGSWSCI in this study differed in terms of mean age, injury level and severity, surgical management and associated injuries. In addition, military and civilian GSWSCI cases differed in terms of gender, mean age, level of injury, surgical management and associated injuries. GSWSCI generally affects young adult males, and, although GSW may not be as common a cause as motor vehicle accident and falls, the consequences may be more devastating in terms of the severity of the injury and associated injuries. The low incidence of tetraplegia is perhaps the only positive aspect of GSWSCI. As the third most common cause of SCI in many countries, GSW can be prevented by more stringent rules for obtaining and carrying firearms, and educational programs.

\section{DATA ARCHIVING}

There were no data to deposit.

\section{CONFLICT OF INTEREST}

The authors declare no conflict of interest.

1 Stefanopoulos PK, Soupiou OT, Pazarakiotis VC, Filippakis K. Wound ballistics of firearm-related injuries-Part 2: Mechanisms of skeletal injury and characteristics of maxillofacial ballistic trauma. Int J Oral Maxillofac Surg 2015; 44: 67-78.

$2 \mathrm{Kim} \mathrm{J}$, Kim JH, Bang MS. A case report of spinal cord injury patient from a high velocity gunshot wound to the lumbar spine. Ann Rehabil Med 2013; 37: 118-122.

3 Bhatoe HS, Singh P. Missile injuries of the spine. Neurol India 2003; 51: 507-511.

4 de Barros Filho TE, Cristante AF, Marcon RM, Ono A, Bilhar R. Gunshot injuries in the spine. Spinal Cord 2014; 52: 504-510.

5 Jaiswal M, Mittal RS. Concept of gunshot wound spine. Asian Spine J 2013; 7: 359-364.

6 Sidhu GS, Ghag A, Prokuski V, Vaccaro AR, Radcliff KE. Civilian gunshot injuries of the spinal cord: a systematic review of the current literature. Clin Orthop Relat Res 2013; 471: 3945-3955 
7 Stefanopoulos PK, Filippakis K, Soupiou OT, Pazarakiotis VC. Wound ballistics of firearm-related injuries-part 1: Missile characteristics and mechanisms of soft tissue wounding. Int J Oral Maxillofac Surg 2014; 43: 1445-1458.

8 Seçer M, Ulutaş M, Yayla E, Cınar K. Upper cervical spinal cord gunshot injury without bone destruction. Int J Surg Case Rep 2014; 5: 149-151.

9 Alaca R, Yilmaz B, Goktepe AS, Yazicioglu K, Gunduz S. Military gunshot wound-induced spinal cord injuries. Mil Med 2002; 167: 926-928.

10 Chen Y, Tang Y, Vogel LC, Devivo MJ. Causes of spinal cord injury. Top Spinal Cord Inj Rehabi 2013; 19: 1-8.

11 Tuğcu I, Tok F, Yılmaz B, Göktepe AS, Alaca R, Yazıcıoğlu K et al. Epidemiologic data of the patients with spinal cord injury: seven years' experience of a single center. Ulus Travma Acil Cerrahi Derg 2011; 17: 533-538.

12 Rahimi-Movaghar V, Sayyah MK, Akbari H, Khorramirouz R, Rasouli MR, Moradi-Lakeh $\mathrm{M}$ et al. Epidemiology of traumatic spinal cord injury in developing countries: a systematic review. Neuroepidemiology 2013; 41: 65-85.
13 Gur A, Kemaloglu MS, Cevik R, Sarac AJ, Nas K, Kapukaya A et al. Characteristics of traumatic spinal cord injuries in south-eastern Anatolia, Turkey: a comparative approach to 10 years' experience. Int J Rehabil Res 2005; 28: 57-62.

14 Kane T, Capen DA, Waters R, Zigler JE, Adkins R. Spinal cord injury from civilian gunshot wounds: the Rancho experience 1980-88. J Spinal Disord 1991; 4: 306-311.

15 Trahan J, Serban D, Tender GC. Gunshot wounds to the spine in post-Katrina New Orleans. Injury 2013; 44: 1601-1606.

16 Burke DA, Linden RD, Zhang YP, Maiste AC, Shields CB. Incidence rates and populations at risk for spinal cord injury: a regional study. Spinal Cord 2001; 39: 274-278.

17 le Roux JC, Dunn RN. Gunshot injuries of the spine-a review of 49 cases managed at the Groote Schuur Acute Spinal Cord Injury Unit. S Afr J Surg 2005; 43 165-168.

18 Bono CM, Heary RF. Gunshot wounds to the spine. Spine J 2004; 4: 230-240. 